\title{
Síndrome de Dolor Inguinal Crónico (SDIC)*
}

\author{
Drs. ALBERTO ACEVEDO F. ${ }^{1}$, JAQUELINE LÓPEZ R. ${ }^{1}$, MARCOS VILLASI M. ${ }^{1}$, \\ AQUILES VITERBO S. ${ }^{1}$, JORGE LEÓN S. ${ }^{1}$
}

\begin{abstract}
Programa de Cirugía Mayor Ambulatoria del CRS Cordillera Oriente. Universidad de Chile, Facultad de Medicina. Santiago, Chile.
\end{abstract}

\begin{abstract}
Chronic inguinal pain syndrome

Background: Chronic inguinal pain syndrome (CIPS) is a frequent complaint in patents attending to a public ambulatory surgical service. Aim: To report a descriptive observational study of CIPS. Material and Methods: Retrospective review of medical records of patients presenting with CIPS between 1999 and 2006. The symptoms and physical examination of the groin, the spine and the coxofemoral joint of these patients was evaluated. Results: CIPS was diagnosed in 150 patients aged 17 to 71 years, 87 men. They represented $14 \%$ of groin problems diagnosed in the study period. Median duration of pain was 13 (3-150) months, with remissions that lasted from weeks to months. The characteristics of pain remained constant in each remission. Pain was present during the daily activities, but it was even worse during resting periods and during night sleep, leading the patient to assume pain reducing positions. Hernia was present in 29 patients and occupied inguinal channel in three. Five patients had a history of previous hernia repair. One hundred twenty patients had spine diseases. An anesthetic blockade of the ilioinguinal nerve was performed in 37 patients with a positive response in seven. Twenty nine of 47 groin ultrasound examinations were informed as abnormal and suggestive of inguinal hernia. Surgical treatment was performed in these patients and was associated with pain abolition in 12, reduction in seven and persistence in the rest. Remaining patients were treated with spine rehabilitation. The short time follow-up of both groups was encouraging. Conclusions: Inguinal hernia and radicular pain due to spine problems are the main causes of CIPS in this series of patients.
\end{abstract}

Key words: Inguinal pain, radicular pain, inguinal hernia.

\section{Resumen}

El Síndrome de Dolor Inguinal Crónico (SDIC) ha sido un hallazgo frecuente en el Centro de Cirugía Mayor Ambulatoria de las Hernias del CRS Cordillera. Presentamos un estudio observacional descriptivo del SDIC, basado en la información contenida en las fichas clínicas en un período de 7 años. Se estudiaron los síntomas, el examen físico de la región inguinal, de la columna vertebral, de la inervación de la zona y de la articulación coxofemoral. El cuadro clínico afectó a 150 pacientes, 87 varones y 63 mujeres, con una edad promedio de 42 años (17-71). La antigüedad de los síntomas fue en promedio de 13 (3-150) meses apreciándose remisiones de duración variable, de semanas o meses, sin variación de las características neuropáticas

*Recibido el 7 de Marzo de 2008 y aceptado para publicación el 19 de Enero de 2009.

Correspondencia: Dr. Alberto Acevedo F.

Las Torres 5100, Peñalolén, Santiago, Chile.

E-mail: aacevedof@gmail.com 
del dolor. Si bien éste se presentó durante la actividad, estuvo presente también en el reposo sentado y el nocturno aliviándose con posición antálgica. Se detectó una hernia inguinal en 29 , un canal inguinal ocupado en 3 y antecedente de herniorrafia en 5 pacientes. En los restantes 129 pacientes se detectó patología de columna vertebral, apreciándose en todos un examen neurológico alterado. El bloqueo del nervio ilioinguinal se efectuó en 37 pacientes siendo positivo en 7. La ecotomografía de partes blandas de la región inguinal adquiere valor sólo en manos experimentadas. Una operación se realizó ante evidencia de patología inguinal, los demás pacientes fueron sometidos a rehabilitación de la columna vertebral. Los resultados a corto plazo han sido satisfactorios en ambos grupos.

Palabras clave: Dolor inguinal crónico, inguinodinia, radiculopatía, neuralgia del nervio ilioinguinal.

\section{Introducción}

El síndrome de dolor inguinal fue descrito por Magee $^{1}$ en 1942, refiriéndose al atrapamiento del nervio génitofemoral en pacientes apendicectomizados o con traumas severos de la región inguinal o del abdomen inferior. Mardsen², destacó, en 1962, la elevada frecuencia con que los pacientes sometidos a una herniorrafia desarrollaban una neuritis del nervio ilioinguinal o del iliohipogástrico, caracterizado por dolor inguinal que él atribuyó al atrapamiento de los nervios en la sutura de la herniorrafia.

Otro cuadro clínico en que el dolor inguinal es el síntoma predominante es aquel que se observa en deportistas de alta competitividad como consecuencia de lesiones músculo esqueléticas ${ }^{3-5}$. Dentro de este grupo de lesiones descritas en atletas se menciona la "sports hernias", producida por una pared posterior del canal inguinal débil y protruyente que afecta los nervios de la zona. Fue Daysine ${ }^{6}$, el primero en destacar que el dolor inguinal crónico puede hacerse presente en la población general sin la presencia de hernia, de antecedentes quirúrgicos o de esfuerzos físicos desmedidos, deportivos o laborales.

En el Consultorio externo del Programa de Cirugía Mayor Ambulatoria de las Hernias del CRS Cordillera de Santiago, el Síndrome de Dolor Inguinal Crónico (SDIC) en sujetos sedentarios estuvo presente en una alta proporción de nuestros pacientes y ello nos llevó a preocuparnos con especial interés del tema, elaborando un protocolo que fue seguido en todos los casos. La presente publicación da cuenta del cuadro clínico del SDIC, su patogenia y nuestros resultados iniciales en su manejo diagnóstico y terapéutico.

\section{Material y Método}

El presente estudio observacional descriptivo, retrospectivo, abarca un período de 6 años, entre el $1^{\circ}$ de Enero de 1999 hasta el 31 de Diciembre de 2006 e incluye a la totalidad de los pacientes que consultaron en el consultorio externo del CRS Cordillera Oriente, derivados de los consultorios de las comunas de Macul y Peñalolén. El examen de los pacientes fue realizado por el jefe del Proyecto de Cirugía Mayor Ambulatoria con la colaboración de los residentes en el espíritu de un trabajo docente asistencial.

La información demográfica, anamnéstica, del examen físico y de los exámenes complementarios, se obtuvo de una ficha preimpresa que se adjuntó a la ficha clínica conteniendo la información pertinente en los pacientes. Esta información se vertió en una base de datos Excel.

En la anamnesis remota se consignaron los antecedentes patológicos provenientes de afección de la columna vertebral, se precisó el antecedente de cirugías previas en el abdomen inferior y se precisó si el paciente realizaba actividades deportivas de alta competitividad o esfuerzos físicos desmedidos.

En lo concerniente al dolor se estudiaron la antigüedad, localización, irradiaciones, periodicidad, la intensidad, influencia del grado de actividad física, frecuencia, el tipo (agudo, subagudo, crónico), el carácter del dolor neuropático (disestésico, neurítico), su presencia durante el reposo nocturno y su alivio mediante posición antálgica. Prescindimos de utilizar EVA para la intensidad del dolor después de comprobar que ello producía gran confusión en nuestros enfermos y lo reemplazamos por una escala de 4 grados (leve, moderado, intenso y muy intenso).

De especial interés fue establecer la presencia de síntomas por compromiso de los nervios cutáneo lateral del muslo (meralgia parestésica), génitofemoral y del nervio femoral, provenientes de raíces vecinas del plexo lumbar (Figuras 1 y 2 ).

$\mathrm{Al}$ examen físico se estudió la región inguinofemoral estableciendo la presencia de una hernia, de un canal inguinal ocupado, de alteraciones del cordón espermático en su trayecto hacia el escroto y de alteraciones del testículo.

El examen de la articulación coxofemoral se efectuó estudiando la movilidad y sensibilidad de la articulación llevando la extremidad a abducción for- 


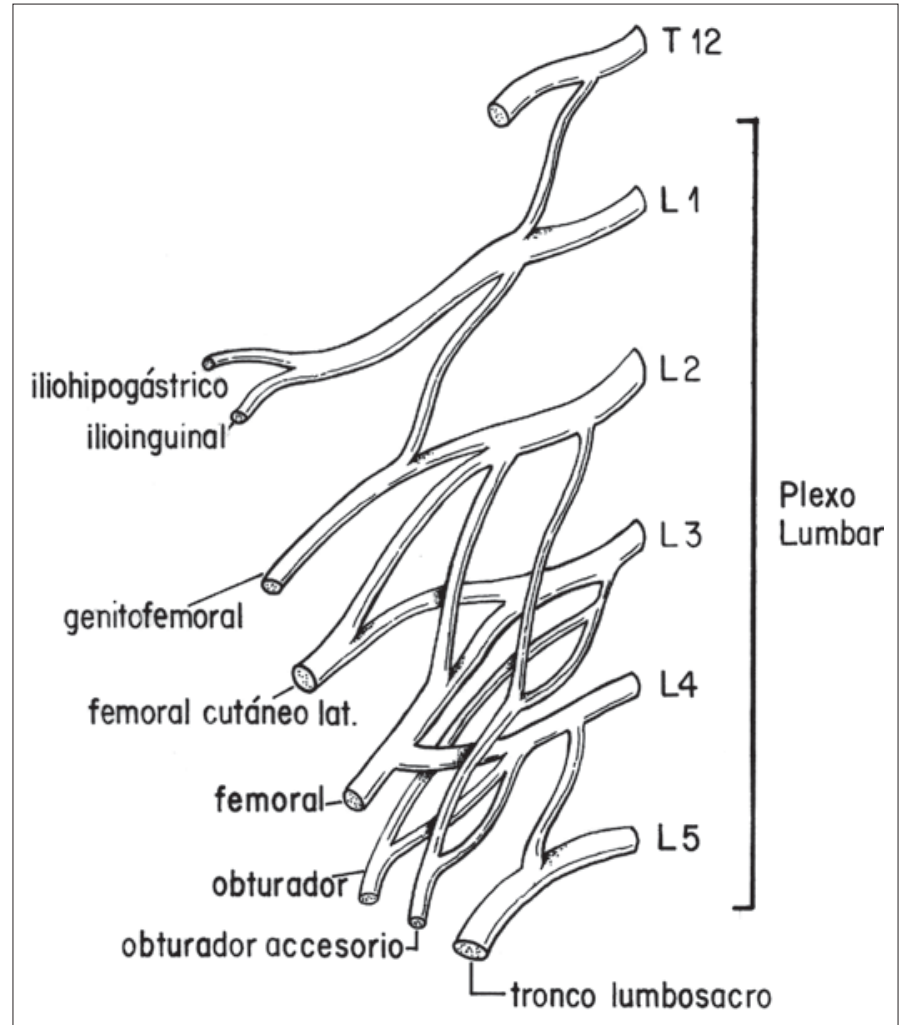

zada, a aducción y a rotación interna y externa, comparando ambos lados.

La columna vertebral se estudió con el paciente de pié. A la inspección se buscaron alteraciones de la forma de la columna (escoliosis, xifosis) así como contracturas de las masas musculares paravertebrales. Se estudió la movilidad a la flexión hacia adelante, hacia los lados y la rotación. Finalmente, se buscó la presencia de dolor a la presión y percusión de las apófisis espinosas y de las masas musculares paravertebrales, buscando puntos precisos dolorosos.

En los pacientes con evidencia de coxartrosis se realizó el estudio radiológico de la articulación coxofemoral.

El estudio radiológico de la columna vertebral antero posterior y en posiciones oblicuas se solicitó inicialmente en forma rutinaria, pero la alta frecuencia de alteraciones a todos los niveles y su inespecificidad nos motivaron a dejar de solicitarlo.

Bloqueo anestésico del nervio ilioinguinal: se efectuó infiltrando con $5 \mathrm{ml}$

Figura 1. Plexo lumbar.

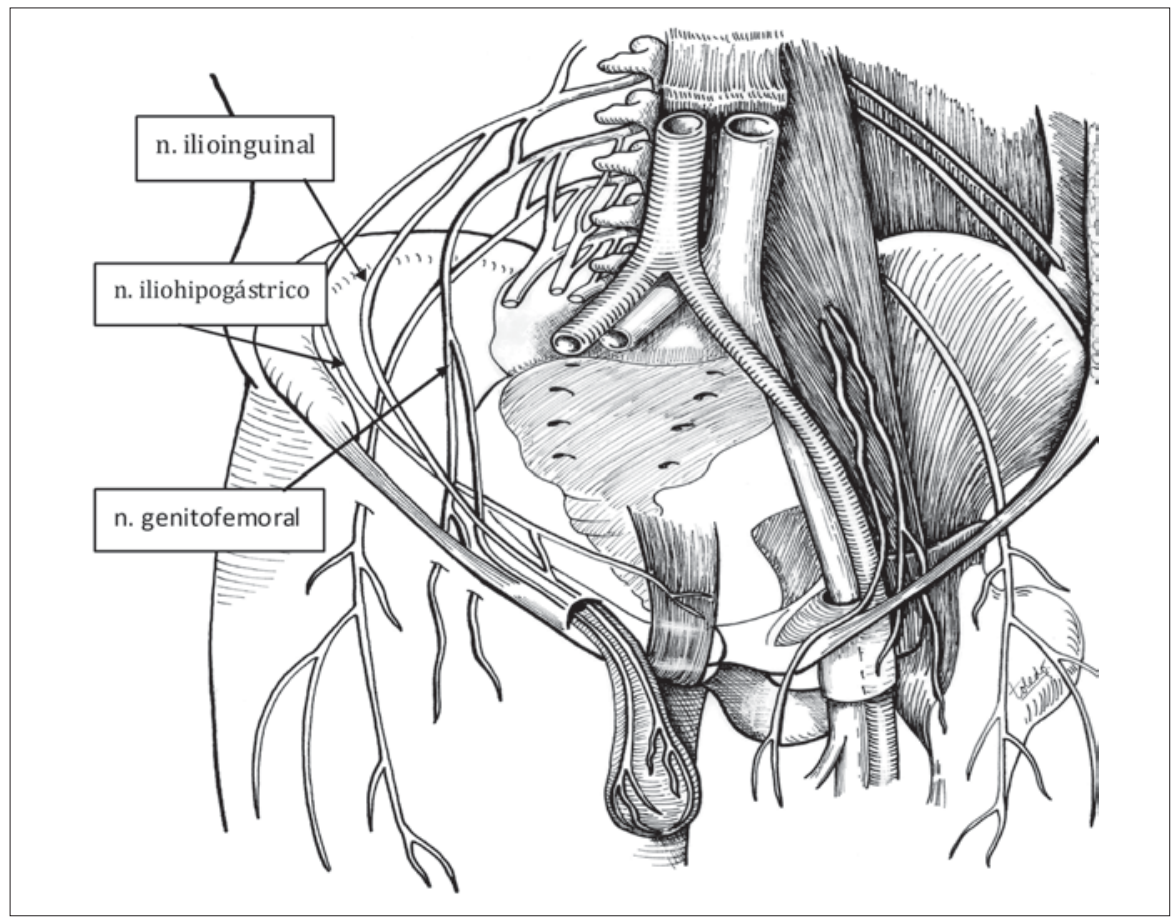

Figura 2. Trayecto de los nervios del plexo lumbar en la pared abdominal. 
de una solución de Lidocaina ${ }^{\circledR}$ al $2 \%$ bajo la aponeurosis del músculo oblicuo externo a la altura de la espina ilíaca anterosuperior. Este examen es sólo practicable cuando existe dolor al momento del examen. La supresión del dolor estaría señalando una afección del nervio en su transcurso por el canal inguinal. Cuando el bloqueo no modificó el dolor asumimos que la lesión estaba localizada proximal al sitio de infiltración.

La ecotomografía de la región inguinal se solicitó en ausencia de patología inguinofemoral, es decir, de una hernia inguinofemoral, de un canal inguinal ocupado y en los pacientes en los que el bloqueo anestésico fue negativo (no abolió el dolor). El resultado del examen fue analizado críticamente ya que es muy operador dependiente.

Los pacientes con indicación quirúrgica se intervinieron efectuándose trineurectomía y tratamiento de la hernia y del defecto de la pared; los pacientes sin indicación quirúrgica fueron derivados a medicina física. Ambos grupos de pacientes fueron controlados por un miembro del staff al cumplir 3 meses de tratamiento. Los resultados fueron anotados en un cuaderno de protocolo.

\section{Resultados}

En el período de tiempo de este estudio concurrieron 2.017 pacientes al consultorio externo de pared abdominal, de los cuales 1.045 fueron hernias de la región inguinofemoral. En este período de tiempo consultaron 150 pacientes con dolor inguinal constituyendo el $7,4 \%$ del total de las consultas y el $14,4 \%$ de las consultas por patología inguinofemoral.

La serie clínica estuvo conformada por 87 varones con una edad promedio de 44 (23-71) años y 63 mujeres con una edad promedio de 41 (17-69) años, que consultaron al policlínico del CRS Cordillera. La relación varón/mujer fue de 1,4/1.

En el grupo no hubo atletas de alto rendimiento, pero un $10 \%$ de los hombres realizaba actividades laborales vinculadas a gran esfuerzo físico.

El dolor en la región inguinal fue el eje sintomático en la totalidad de los enfermos y estuvo ligado a una impotencia funcional (laboral) en 93 casos (62\%). La discapacidad fue leve en 45, moderada en 37 y severa en 11 oportunidades. La antigüedad de los síntomas fue en promedio de 13 (3-120) meses.

El dolor fue periódico remitiendo por períodos de semanas o meses, conservando las mismas características en cada una de las recidivas. En los períodos sintomáticos el dolor fue calificado como permanente con exacerbaciones por el $12 \%$, frecuen- te (más de una vez al día) en el $63 \%$ y ocasional (más de una vez a la semana) en el $25 \%$ de los casos.

En los períodos sintomáticos la intensidad fue variable; fue calificada como leve por el $18 \%$, como moderada por el $65 \%$ y como intensa por el $17 \%$ de los pacientes.

La gran mayoría de los enfermos refirió exacerbación del dolor al estar de pie o al realizar sus actividades habituales. En el $92 \%$ de los casos el dolor se presentó en el reposo, tanto en posición sentada como durante el reposo nocturno asociándose a la búsqueda de una posición antálgica en el $88 \%$ de los casos.

El dolor fue subagudo con exacerbaciones, no apreciándose formas agudas ni crónicas. El carácter del dolor fue neuropático en todos los casos. Fue predominantemente disestésico (urente, parestésico) en el $85 \%$ y predominantemente neurítico (punzante, lancinante) en el 15\% de los casos.

El examen físico de la región inguinal permitió establecer la presencia de una hernia inguinal en 23 pacientes, una herniorrafia previa en 5 , un canal inguinal ocupado en 3 y una región inguinofemoral normal en 119 oportunidades.

La columna vertebral estuvo alterada en 129 pacientes: deformidad en 35 , rigidez y movilidad alterada en 42 , contractura de masas musculares paravertebrales en 28 y percusión dolorosa a nivel de D-12 L-1 en 128 casos.

El estudio de la sensibilidad que efectuamos durante la primera consulta raspando y pinchando con una aguja hipodérmica y tocando mediante torunda de algodón estuvo alterado en 134 pacientes.

El examen de la articulación coxofemoral estuvo alterado en 3 pacientes. La radiología confirmó diversos grados de artrosis.

La ecotomografía de la región inguinal se solicitó o fue proporcionada por el paciente en su primera consulta en 47 oportunidades. El examen fue informado como alterado y sugerente de una hernia inguinal en 29 ocasiones. La visualización del anillo herniario sólo se consiguió en 4 oportunidades y la del saco herniario con aumento de volumen con la maniobra de Valsava estuvo presente en los 29 pacientes.

El bloqueo anestésico del nervio ilioinguinal se efectuó en 35 oportunidades. La abolición del dolor se produjo en 4 pacientes y en otros 3 fue dudosa. En estos pacientes se efectuó una exploración de la región inguinal, una trineurectomía y una corrección de las alteraciones anatomopatológicas presentes.

El diagnóstico de hernia inguinal y de canal inguinal ocupado se corroboró durante el tratamiento quirúrgico. En todos los casos se exploró el ca- 
nal inguinal, se efectuó trineurectomía y se realizó herniorrafia. El seguimiento efectuado a los 3 meses de la cirugía en estos pacientes comprobó abolición del dolor en 12 pacientes, disminución de éste en 7 y persistencia de las molestias en el resto.

Los pacientes sometidos a rehabilitación de la columna vertebral experimentaron una desaparición o mejoría franca de sus molestias. Sólo un paciente fue derivado a neurocirugía por persistencia de sus molestias a pesar de la kinesiterapia bien realizada. Intervenido de una hernia del núcleo pulposo a nivel de D12 L1, se terminó el dolor.

\section{Discusión}

En 2002, Deysine ${ }^{6}$ llamó la atención sobre el síndrome de dolor inguinal sin la presencia de una hernia, y sin el antecedente de una herniorrafia previa. El autor estableció que la patogenia de este cuadro es, en la mayoría de los casos, una radiculopatía de las raíces D12-L1 (Figura 1) o una neuropatía que afecta a los nervios ilioinguinal, iliohipogástrico o a la rama genital del nervio génitofemoral (Figura 2).

Estos pacientes son frecuentemente derivados al cirujano con el diagnóstico de una hernia inguinal y por ello es necesario, cuando hay una hernia, que el cirujano reconozca el SDIC e informe al paciente, ya que de no hacerlo arriesga a que la persistencia del dolor en el postoperatorio sea atribuida a la acción quirúrgica.

El dolor neuropático se debe a una lesión nerviosa central o periférica. Tiene un componente basal, urente, continuo, de intensidad variable, y exacerbaciones agudas, paroxísticas, lancinantes, breves denominadas neuríticas. Lo habitual es que la sensación urente, continua y las sensaciones lancinantes coexistan asociadas a parestesias y disestesias $^{9,10}$. La obtención del carácter del dolor suele ser difícil y requiere dedicación por parte del médico y paciencia por parte del enfermo. La presencia de estigmas de daño neurológico (radiculopatías, neuralgias) y de síntomas de patología de la columna vertebral ayudará al diagnóstico.

El presente estudio establece que las características del dolor referido por los pacientes con el SDIC difieren claramente de los referidos por los pacientes portadores de hernias de la región inguino-

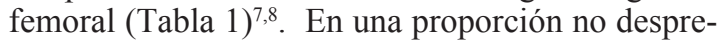
ciable de pacientes el cirujano comprobará una hernia inguinofemoral o un canal inguinal ocupado y tenderá a atribuir el dolor a éstas. Una anamnesis cuidadosa permitirá, sin embargo, comprobar que el dolor difiere del que aqueja a los pacientes con hernia.
Tabla 1. Características que diferencian a las hernias inguinales del SDIC

\begin{tabular}{lcc}
\hline & Hernia & SDIC \\
\hline Relación hombre/mujer & $7 / 1$ & $3 / 2$ \\
Edad & $56,2(15-88)$ & $43,4(17-71)$ \\
Dolor & Nociceptivo & Neuropático \\
Periodicidad & Ausente & Presente \\
Desaparece con el reposo & Sí & No \\
Dolor nocturno & Sí & No \\
Posición antálgica & Sí & No \\
Meralgia parestésica & Ausente & Presente \\
Patología de columna & Ausente & Presente \\
\hline
\end{tabular}

Al examen físico lo primero que llama la atención del cirujano es la ausencia de una hernia inguinal y en estas circunstancias es imperativo dirigir la atención a la columna vertebral. Hemos dado especial importancia a la percusión de los cuerpos vertebrales y de las masas musculares paravertebrales con un golpe de puño. El dolor a la percusión a nivel de los cuerpos vertebrales DX-XII y LI estuvo presente en el 85,3\% de los pacientes con SDIC.

La sensibilidad táctil en la región inguinal está con frecuencia alterada, sin embargo, se trata de información subjetiva difícil de reproducir, y por ello la realizamos excepcionalmente.

La Figura 3 muestra en diagramas de flujo nuestra actual conducta en los pacientes con SDIC sin patología inguinal evidente. Es necesario destacar la importancia que le damos al bloqueo del nervio ilioinguinal. Si el bloqueo anestésico hace desaparecer el dolor, deducimos que el daño neurológico es periférico y asumimos que hay una alteración en el trayecto del nervio dentro del canal inguinal. Cuando se obtiene este resultado procedemos a la intervención. Si el bloqueo anestésico es negativo tenemos la opción de enviarlo directamente a Medicina Física para la rehabilitación de la columna. El tratamiento fisioterapéutico de la columna vertebral adquiere el carácter de una prueba diagnóstica, ya que de ser exitoso permite localizar el sitio de origen del daño a nivel radicular.

La ecotomografía de partes blandas de la región inguinal es muy operador dependiente y por ello hemos usado esta opción sólo si el radiólogo tiene experiencia en el examen de los tejidos blandos del abdomen.

El diagnóstico diferencial del SDIC debe hacerse con el dolor inguinal en atletas. Éste se presenta en deportistas profesionales y de elite. La palpación y compresión de las prominencias óseas así como el 


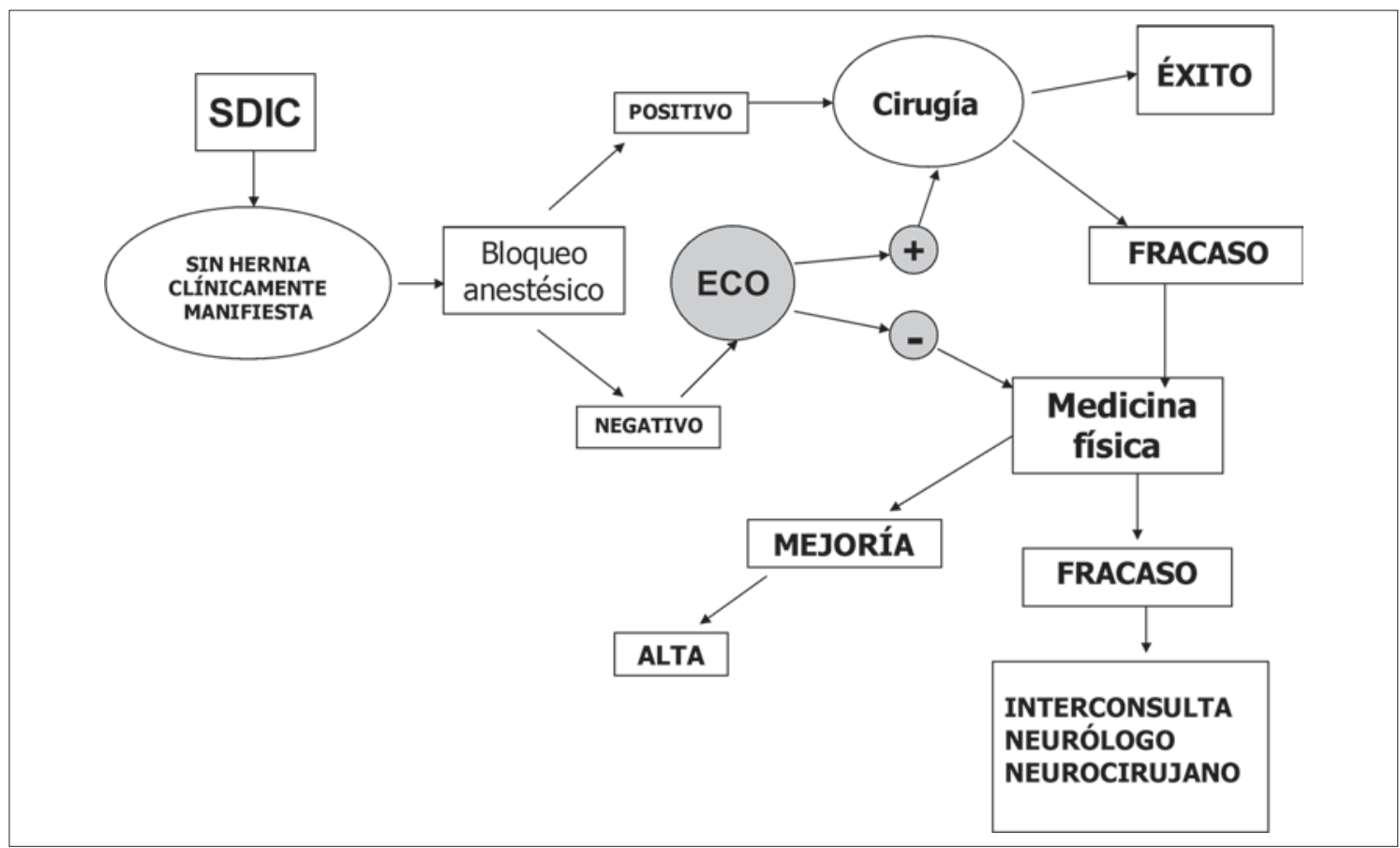

Figura 3. Diagrama de flujo diagnóstico y terapéutico del SDIC en ausencia de una hernia.

estudio de la motilidad ayudará al diagnóstico al producir una exacerbación del dolor ${ }^{3-5}$. No siempre es posible determinar la etiología del dolor inguinal que aflige a los atletas y para estos casos, Gilmore ${ }^{11}$, presenta el concepto de "groin disruption" que podríamos traducir como desgarro o ruptura de diversas estructuras del canal inguinal, pero que no constituyen una hernia.

El estudio radiológico con herniografía en pacientes con dolor inguinal ha permitido reconocer lo que se ha llamado hernias ocultas, las que se darían principalmente en mujeres obesas y evidenciaría, principalmente, hernias pequeñas, femorales y obturatrices ${ }^{12}$. Por el mismo método se han descrito alteraciones de la pared posterior del canal inguinal en los atletas con dolor inguinal. Estos hallazgos han dado origen al "syndrome of broad and deep fossae" de la pared posterior del canal inguinal ${ }^{13}$.

El tratamiento de los enfermos con SDIC asociados a una hernia inguinal o a un canal inguinal ocupado es quirúrgico y así hemos procedido. El resultado inicial, con seguimiento a 3 meses ha sido satisfactorio en el 58\% de los casos. En el resto, el dolor ha persistido más allá de este período de tiempo. Estos pacientes han sido derivados a medicina física con el diagnóstico de una radiculopatía. La información que damos a nuestros enfermos con hernia sobre la coexistencia de ésta con el SDIC ha contribuido a evitar problemas legales.

En los pacientes derivados al fisiatra para una rehabilitación de la columna vertebral, el control efectuado a los 3 meses mostró la desaparición o disminución acentuada del dolor en la gran mayoría de los enfermos. Sólo excepcionalmente y ante el fracaso de la rehabilitación los pacientes son derivados al neurocirujano para efectuar un examen exhaustivo de la columna y proceder eventualmente a la corrección quirúrgica de las lesiones.

De entre las escasas publicaciones sobre el dolor inguinal crónico destacan las de Lee y Dellon $^{14,15}$, quienes establecen que la anamnesis y el examen físico bastarían para diferenciar el nervio afectado en los pacientes con dolor inguinal crónico. El autor propone intervenir quirúrgicamente estos pacientes buscando eliminar la causa del atrapamiento o daño del nervio con resultados satisfactorios sobretodo en la neuralgia del nervio ilioinguinal.

La mayor atención de los cirujanos se ha dirigido al cuadro del dolor inguinal crónico secundario a herniorrafia inguinofemoral y existe un gran número de publicaciones que tratan el tema desde diferentes perspectivas ${ }^{16,17}$. Debemos destacar, sin embargo, que el SDIC debe ser distinguido de la neuralgia postherniorrafia ya que se trata en la ma- 
yoría de los casos de una afección neurológica desvinculada de la presencia o ausencia de una hernia primaria o recidivada.

\section{Referencias}

1. Magee RK. Genitofemoral causalgia (a new syndrome) Can Med Assoc J 1942; 46: 326-329.

2. Mardsen AJ. Ilioinguinal hernia: a three-year review of two thousand cases. Br J Surg 1962; 49: 384-394.

3. Morelli V, Smith, V. Groin injuries in athletes. Am Fam Physician 2002; 64: 1405-1414.

4. Westlin N. Groin pain in athletes from southern Sweden. Sports Med Arthroscopy Rev 1997; 5: 280284.

5. Meyers WC, Foley AP, Garrett VE, Lohnes JH, Mandlebaum BR. Management of severe lower abdominal or inguinal pain in high performance athletes. Am J Sports Med 2000; 28: 2-8.

6. Deysine M, Deysine GR, Reed JR. Groin pain in the absence of hernia: a new syndrome. Hernia 2002; 6: 64-67.

7. Acevedo A, Viterbo A, Arriaza N, Valenzuela L, Revello L, Díaz F. Síndrome de dolor inguinal crónico (SDIC), un síndrome interdisciplinario. Rev Chil Cir 2007; 59, (Supl): 122.

8. Acevedo A, Viterbo A, Cápona R, Dellepiane V. Manifestaciones clínicas de la hernia inguinal. ¿Qué mo- tiva al paciente a buscar una solución quirúrgica? Rev Chil Cir 2007: 59, (Supl): 125.

9. Gales BS, Jensen MP. Development and clinical validation of a pain measure specific to neuropatic pain. Neurology 1997; 48: 332-338.

10. Baron R. Peripheral neuropatic pain from mechanisms to symptoms. Clin J Pain 2000: 16: 512-520.

11. Gilmore OJ. Groin disruption in sportsmen. En Kurzer M, Kark AE, Wanz GE. Surgical management of abdominal wall hernias. Londres: Martin Dunnitz, 1999: 151-157.

12. Hamlin JA, Kahn AM. Herniography: a revue of 333 herniograms. American Surgeon 1998; 64: 965-969.

13. Hackney RG. The sports hernia: a cause of chronic groin pain. British J of Sports Med 1993; 27: 58-61.

14. Lee Ch, Dellon AL. Surgical management of groin pain of neural origin. J Am Coll Surg 2000; 191: 137142.

15. Nahabedian MY, Dellon AL. Outcome of operative management of nerve injuries in the ilioinguinal region. J Am Coll Surg 1997; 184: 265-268.

16. Vrijland WW, van den Tol MP, Luijendijk RW, Hop WC, Busschbach JJ. Randomized clinical trial of non mesh versus mesh repair o primary inguinal hernia. $\mathrm{Br}$ J Surg 2002; 89: 293-297.

17. Nienhuijs S, Staal E, Strobbe L, Rosean C, Groenewoud H. Chronic pain alter mesh repair of inguinal hernia: a systemic review. Am J Surg 2007; 1994: 394-400. 